\title{
El análisis de las violencias en contra de las mujeres: una aproximación a la teoría feminista
}

\author{
The analysis of violence against women: an approach to feminist theory \\ Karla María Zúñiga Santillán ${ }^{a}$, Araceli Jiménez-Pelcastre ${ }^{b}$
}

\begin{abstract}
:
This essay aims to show that the study of gender violence, specifically the violence against women, has a solid theoretical basis, which reveals the relations of domination and identity constructions between women and men present in the social structure in which we live. As a starting point, feminist epistemologies allow a different perspective to the traditional theories of the social sciences. Compatible with them, the gender analytical category and the concept of structural violence allow us to analyze the ways in which violence against women is reproduced, legitimized and naturalized.
\end{abstract}

\section{Keywords:}

Feminist epistemology. Gender. Domination. Structural violence.

\section{Resumen:}

Este ensayo tiene por objetivo mostrar que, para el estudio de las violencias de género, en específico las violencias en contra de las mujeres, existe una base teórica sólida, la cual devela las relaciones de dominación y las construcciones de la identidad entre mujeres y hombres presentes en la estructura social en la que vivimos. Como punto de partida, las epistemologías feministas permiten una perspectiva distinta a las teorías tradicionales de las ciencias sociales. Compatible con ellas, la categoría analítica de género y el concepto de violencia estructural permiten analizar las formas en que se reproducen, legitiman y naturalizan las violencias en contra de las mujeres.

\section{Palabras Clave:}

Epistemología feminista. Género. Dominación. Violencia estructural.

\footnotetext{
a Estudiante de la Maestría en Ciencias Sociales, Instituto de Ciencias Sociales y Humanidades, Universidad Autónoma del Estado de Hidalgo, Pachuca, México. e-mail: karla.zsantillan@gmail.com

${ }^{\mathrm{b}}$ Profesora-investigadora en el Instituto de Ciencias Sociales y Humanidades, Universidad Autónoma del Estado de Hidalgo, Pachuca, México. ORCID: 0000-0002-4043-5485.e-mail: aracelijip@ hotmail.com
} 


\section{Introducción}

Abordar las violencias de género ejercidas contra las mujeres desde un marco teórico y contextual fundamentado es necesario, para construir conocimientos que conlleven al acercamiento de las realidades que nos pertenecen. En principio, debe quedar claro nuestro posicionamiento al hablar de múltiples violencias y en este sentido considerar que, en la normalización de los géneros, se construyen relaciones generizadas. Estas relaciones se anclan en las diferencias, que a su vez detonan desigualdades, discriminaciones y desventajas o privilegios adquiridos con base en poderes y derechos dados a partir del género (Biglia, 2007). El género, no opera aisladamente, existen otras múltiples categorías vinculadas entre sí de diversas maneras, que determinan la posición individual o grupal dentro la sociedad; algunas de ellas son: la clase social, la etnia, la orientación sexual y la edad, elementos que al interseccionarse (Meza, 2018) permiten considerar y reconocer a las violencias de género, en su pluralidad y complejidad.

En los procesos de investigación sobre las violencias, nos situamos frente a esas otras miradas que reflejan y nos dan voz como mujeres, no solo como sujetas de estudio, sino también como sujetas de acción en realidades tan cotidianas y por lo mismo, tan habituales, que se camuflan en el diario acontecer, y pocas veces nos detenemos a pensar en las implicaciones sociales, económicas y políticas de su existencia. Las violencias son un problema sumamente complejo, por tal razón, solamente nos centramos en un ámbito en el que, como señala Sandra Harding (2002, p: 18) "si se toman en cuenta las estadísticas sobre violencia contra las mujeres, resulta razonable situar a la mayoría de las culturas contemporáneas entre las más salvajes de todos los tiempos".

Nuestra intención es aportar nociones para abordar el tema de las violencias contra las mujeres desde el marco específico de las epistemologías feministas (Harding, 1996). Frente a otras epistemologías que orientan para realizar investigaciones, nos decantamos por las que surgieron de las afirmaciones $y$ preocupaciones feministas llevadas a cabo en torno a movimientos políticos implicados con el cambio social de las mujeres, porque permiten una valoración plural de las múltiples y distintas maneras en que las realidades pueden ser entendidas y la manera en cómo construir el conocimiento.

En este sentido, este acercamiento podría también interpretarse como parte de una reivindicación de las luchas feministas iniciadas desde el siglo XVIII y fortalecidas durante el siglo $\mathrm{XX}$ en pro de los derechos humanos de las mujeres. Este trabajo continuo, ha servido tanto para lograr el reconocimiento de las mujeres fuera del ámbito privado, como para señalar las formas de subordinación-dominación que han sido parte de nuestras relaciones sociales e históricas y para enmarcar las miradas críticas hacia las violencias. Señalando la necesidad de visibilizar las representaciones que reproducen simbólica 0 literalmente las desigualdades entre hombres y mujeres $\mathrm{y}$, además, resignificar nuestro papel fuera de las tradicionales construcciones sociales y culturales del género.

Abordar el tema de las violencias contra las mujeres, desde el marco específico de las epistemologías feministas permite explicar y ubicar las categorías que influyen para que este concepto sea problematizado como un fenómeno y problema social. De esta manera, es posible el reconocimiento, la comprensión y la apropiación de las realidades que deben mirarse bajo una visión crítica. Siguiendo la caracterización de Harding (1996), las epistemologías feministas aglutinan al feminismo empirista, el punto de vista feminista y el posmodernismo feminista. Será el punto de vista feminista el que nos aproxime a definir cómo emprender investigaciones sobre el tema en cuestión.

Las epistemologías feministas, buscan construir un conocimiento que pueda estar fuera del androcentrismo o falogocentrismo (Araiza, 2012) y más cercano a la realidad de las mujeres. Si bien las ciencias sociales centran el estudio en los seres humanos como sujetos de conocimiento y su relación con el entorno y contexto del que somos parte, no debemos olvidar que las violencias de género requieren inevitablemente posicionarnos desde otras alternativas que expliquen este fenómeno. Esas alternativas se encuentran vinculadas a los marcos propuestos en los estudios feministas, mismos que han permitido conceptualizar entre otros, el género, la dominación y la violencia estructural, como claves teóricas fundamentales. La descripción de la teoría del punto de vista feminista, la categoría de género y la violencia estructural como referentes para el estudio de las violencias, son los apartados que contiene el presente ensayo.

\section{Una aproximación epistemológica feminista para el estudio de las violencias contra las mujeres}

Antes de entrar en materia de discusión sobre la pertinencia de la teoría del punto de vista feminista, para el estudio de las violencias contra las mujeres, aclararemos algunas nociones que son necesarias. Reconociendo que las violencias son acciones, consecuencia de la complejidad de las relaciones sociales, es importante advertir sobre la existencia de sesgos y errores en torno a otros conceptos y teorías 
con los que se estudia el tema (European Commission, 2011), con el afán de realizar reformulaciones en los fundamentos.

No se trata solamente de hacer una crítica radical a la teoría tradicional o positivista de hacer ciencia, sino al contrario, reflexionar con respecto a las implicaciones de esa visión paradigmática y sus planteamientos, que proponen observar y cuidar detalladamente los hechos, con la finalidad de evitar un alejamiento o una visión poco objetiva en las investigaciones, porque tal escisión entre lo objetivo y lo subjetivo hace precisamente que los análisis sobre las violencias en contra de las mujeres queden incompletos si solamente se considera el punto de vista de quien hace la investigación.

Por otro lado, es importante señalar, que los estudios que utilizan enfoques propios de las epistemologías feministas, reconocen la importancia de retomar al género como categoría analítica, cuya implicación es importante en el estudio de las violencias en contra de las mujeres. En este sentido, abordar la interseccionalidad es también un referente indispensable en los estudios de género, al entenderse como un concepto que se encuentra en interacción constante con diversas categorías de análisis: edad, clase, etnia, etc., para una mayor comprensión del fenómeno, debido a que tanto en lo colectivo como en lo individual determinan el lugar que cada persona ocupa dentro de la sociedad (Blazquez, 2012). En otras palabras, las intersecciones que resultan, determinan la variedad y la existencia de mayores ventajas o desventajas, para una persona $u$ otra $y$, por tanto, los niveles de desigualdad que subyacen frente a los distintos sistemas de opresión (Meza, 2018).

Por tal motivo, los planteamientos expuestos implican considerar contextualmente las relaciones que se construyen en la vida de las mujeres. En el caso particular de las violencias, se trata de relaciones entre mujeres y hombres; y es en el discurso y las prácticas donde se reproducen estas relaciones de dominaciónsubordinación. Las situaciones violentas ocurren en todos los ámbitos en los que las mujeres nos desarrollamos: familiar, laboral, escolar, institucional, comunitario y comprenden también diversos tipos, abarcando afectaciones en lo físico, psicológico, económico, sexual, patrimonial, así como la violencia feminicida considerada una forma extrema de violencia de género contra las mujeres (DOF, 2007). Precisamente, las epistemologías feministas proponen adentrarnos con profundidad en esas realidades para responder a las preguntas sobre cómo y por qué se dan las dicotomías de poder que legitiman las violencias de género.

Las epistemologías feministas tienen antecedentes que es necesario mostrar. Marcela Lagarde (2013) refiere que el término feminismo define a una corriente crítica que reconoce el orden patriarcal, la condición de género y por tanto, las relaciones de desigualdad entre mujeres y hombres, que se enmarcan dentro de un momento histórico y cultural determinado. Los feminismos anclan sus raíces en los movimientos surgidos en el siglo XVIII, que lucharon por los derechos e igualdad de condiciones para las mujeres, frente a las prerrogativas de las que ya gozaban los hombres en tanto eran reconocidos como ciudadanos. Los movimientos feministas denunciaban inicialmente que, la casi generalidad de las mujeres, estaban sometidas al ámbito privado, limitadas a vivir y tener un desarrollo valorativo y social meramente reproductivo, dentro de los márgenes de los hogares, siendo situaciones establecidas socioculturalmente (Amorós y De Miguel, 2005).

Durante la segunda mitad del siglo XX las motivaciones de los movimientos feministas pasaron a formar parte de las preocupaciones e investigaciones entre los círculos académicos de las universidades, generando un amplio campo de conocimientos, que posteriormente se reconocieron como científicos dentro de las ciencias sociales (Beltrán y Maquieira, 2001). Así, dada la necesidad de contar con un marco conceptual que fuera utilizado como referente en este tipo de investigaciones, se comenzaron a producir ideas que abrieron los marcos hasta entonces establecidos y definieron nociones propias para el estudio de las cuestiones que afectan a las mujeres. Entre muchos otros, es de trascendencia el concepto de género y las epistemologías feministas.

Las epistemologías feministas generaron una discusión en el campo del conocimiento, al proponer la posibilidad de enfocar a las y los sujetos de las investigaciones fuera de las miradas tradicionales, que presuponen la existencia de verdades producidas dentro de las nociones androcentristas de hacer ciencia, cuyos criterios de objetividad refuerzan la dominación masculina en el conocimiento, situación que se conoce como falogocentrismo (Araiza, 2012). Tradicionalmente las mujeres, como sujetas de investigación, no éramos tomadas en cuenta, motivo de la exclusión prácticamente naturalizada por parte de las ciencias sociales (Harding, 2002). Las epistemologías feministas entonces, pretenden reconocer la subjetividad, que es en donde se encuentra la riqueza de las investigaciones sociales, por tal razón proponen indagar en las experiencias cotidianas y los contextos donde nos movemos las mujeres, utilizando una mirada profunda y horizontal, para dar cuenta que la vida de las personas concretas está condicionada por las relaciones sociales. Con respecto a la subjetividad, la teoría feminista plantea que es sumamente importante ir hacia el estudio de las vidas de las mujeres y por ende, de las violencias, 
considerando el contexto y las experiencias particulares, donde además del género están en interacción muchos otros elementos como la etnia, la clase, la edad y la preferencia sexual (Blázquez, 2012). Estos argumentos implican una ruptura con la forma de hacer investigaciones regidas por criterios positivistas y se dirigen a mirar con profundidad aspectos que no son precisamente cuantificables, como los valores, las emociones, las experiencias y las proyecciones individuales.

En el sentido de justificar las afirmaciones que las epistemologías feministas se proponen, pueden señalarse tres perspectivas, que surgen dentro de los debates críticos y de reflexión sobre la manera de hacer ciencia: el empirismo feminista, el punto de vista feminista y el postmodernismo feminista (Harding, 1996).

Para Sandra Harding (1996), el empirismo feminista se basa principalmente en abonar a la metodología científica una perspectiva fuera de las nociones sexistas y androcentristas que reproducen sesgos. Esto significa que se toma como fuente de objetividad la presencia misma de un grupo feminista dentro del campo científico para llevar a cabo las investigaciones. La afirmación de esta perspectiva se basa en la idea de trabajar desde normas científicas y metodológicas que no solo se apliquen al contexto de la justificación, sino que de igual forma se encuentren presentes en la identificación del problema a investigar. Aún con sus debates internos entre la neutralidad de la ciencia y el posicionamiento respecto al carácter metodológico y normativo; este enfoque tiene su mayor aporte al considerar a las comunidades científicas como espacios en donde se producen los conocimientos y reconoce que los grupos con mayor diversidad de opiniones son los que mayores argumentos válidos pueden aportar, interactuando entre sí y construyendo razonamientos más colaborativos que permitan contrastar las múltiples formas de pensamiento, sus argumentaciones y las conclusiones a las que arriban (Araiza, 2017).

El punto de vista feminista, es otra de las formas abordadas por Harding (1996). Este enfoque plantea la idea de desarrollar el conocimiento científico desde la posición menos privilegiada que históricamente se nos ha asignado a las mujeres, ya que solo de esta manera se puede tener acceso a un conocimiento más real y completo, instalado dentro de las transformaciones que han realizado los movimientos de las mujeres como lucha política. Se trata, en todo momento, de trabajar desde nuestras interpretaciones y experiencias de la vida social, es decir, desde nuestro "punto de vista", elemento que está determinado por el contexto histórico. Con ello se busca superar los conocimientos parciales y hegemónicos que se producen bajo la óptica dominante de hacer ciencia.

Por último, el postmodernismo feminista, se desarrolla en torno a una discusión y crítica respecto a las dos corrientes anteriores, propone una investigación desde las diversas identidades de las mujeres y su fragmentación con otras categorías como raza, etnia, clase, etc. El posmodernismo feminista analiza los esencialismos y la naturalización que incluso en el feminismo han surgido con relación a la identidad "humana", y supone que solo puede existir una verdadera reivindicación feminista, si se toman en consideración todas las identidades fragmentadas con las implicaciones políticas que se crean alrededor de las mismas (Harding, 1996).

A partir del acercamiento teórico enunciado, consideramos pertinente el punto de vista feminista, como posicionamiento epistémico para el estudio de las violencias contra las mujeres, porque recomienda utilizar la mirada reflexiva de las mujeres como sujetas de conocimiento entre los grupos y espacios que nos pertenecen, así como en los que somos subordinadas, analizando los márgenes culturales y de microinteracciones que promueven un orden social de dominación y desigualdad. Conviene enfatizar que la corriente del punto de vista feminista mantiene la intención política de los movimientos feministas, es decir que busca transformar las realidades sociales que nos afectan, como es el caso de las violencias y señala también, que las mujeres debemos abandonar los márgenes para colocarnos en el centro, a la hora de construir conocimientos.

En este sentido, para investigar sobre las violencias que han experimentado otras mujeres se requiere revisar las propias experiencias vividas y la manera en que son entendidas por nosotras. Este paso del proceso permitirá, en consecuencia, estudiar y visibilizar las experiencias de las demás mujeres, precisar sus demandas y ofrecer vías para que emerjan de las situaciones que han padecido. En general, "el feminismo del punto de vista plantea una reflexión y una transformación del conocimiento a partir de su metodología más cercana a lo político, o al menos más consciente de ello y con otras posibilidades más subversivas" (Araiza, 2017:96).

Estas concepciones han permitido que epistemológicamente quienes realizan las investigaciones jueguen también un papel político, para dar voz y volver agentes a las mujeres como sujetas de conocimiento. El afán puesto en construir una ciencia no androcéntrica, permite incluir esas otras miradas de personas históricamente silenciadas, proporcionando a los conocimientos resultantes un privilegio epistémico cercano al fin político y ético del feminismo, que es 
transformar la sociedad y sus sistemas de opresión (Araiza, 2012).

En los sistemas donde imperan las relaciones de poder y por ende las violencias, las mujeres resultamos ser las más afectadas (Maddo y Niebrugge-Branlley, 1997). Precisamente es en esos temas donde las epistemologías feministas, y concretamente el punto de vista feminista, se cuestionan sobre la manera en que se han construido y validado los conocimientos para ser considerados científicos, desarrollando como uno de los intereses de sus estudios lo que ocurre con las víctimas de la dominación masculina. Sandra Harding (2002) indica que es dentro de este campo, el de la dominación, donde se puede profundizar y justificar el estudio de las violencias contra las mujeres. Sin embargo, aún en este tema y en todos los relacionados con la dominación, hay sesgos que deben ser señalados y conocimientos que deben ser deconstruídos, tal es el caso de los conceptos y prácticas que revictimizan nuestro papel subordinado en el caso específico de las violencias.

Para Harding (2002) es incorrecto poner en una posición de sumisión absoluta a las mujeres, por el contrario, se nos debe concebir como personas con capacidad de agencia, que en el plano colectivo hemos actuado en pro de nuestros derechos y su visibilización. Por otro lado, es preciso considerar que aún en las relaciones más brutales de dominación, existen procesos de resistencia y justo es ahí, donde las epistemologías feministas y particularmente el punto de vista feminista enmarca los estudios, porque se trata de incluir otras percepciones que complementan las indagaciones en torno al problema social en cuestión (Bartra, 2012), para trascender los conocimientos construidos únicamente por la o las personas que realizan las investigaciones; situación que es recurrente en las otras formas de hacer investigación, que operan bajo criterios estrictos de objetividad, neutralidad y universalidad.

En clave feminista, resulta fundamental tener una línea de seguimiento que posibilite la comprensión de las relaciones de género en su totalidad, comenzando con las construcciones que cada persona ha hecho a lo largo de su vida y cómo han influido en sus relaciones con otras mujeres y otros hombres, de esta manera se pueden llegar a definir las experiencias violentas que han tenido. Se trata de no perder de vista que las mujeres que vivimos violencias ocupamos un lugar en el universo de relaciones, con una compleja carga subjetiva, hacia la que se debe mirar de manera focalizada y contextualizada, para comprender la diversidad de experiencias de vida en relación al tiempo y espacio en el que nos desenvolvemos y sobrevivimos. Ante esto, es importante que en todo momento tomemos en cuenta la producción y validación de los conocimientos científicos construidos bajo esta óptica, así como su influencia en el campo de la investigación. Precisamente, el punto de vista feminista facilita identificar las teorías y construcciones que en el campo científico siguen manteniendo una posición de desventaja y que por razón de género o cualquier otra subjetividad han estado en los márgenes de lo que se considera como ciencia. De no hacerlo, se refuerzan las jerarquías de género existentes (Martín, 2008).

Abordar las violencias contra las mujeres bajo esta óptica, presenta entonces un reto teórico, pues implica poner en discusión que se trata de un problema social que afecta directamente a un colectivo al que se ha oprimido históricamente. Dicha opresión se encuentra sustentada por la cuestión biológica y deviene en la imposición del poder sobre los cuerpos de las mujeres. En consecuencia, desde el primer acercamiento a los planteamientos para desarrollar investigaciones sobre las violencias en contra de las mujeres, es preciso conocer las debilidades o los aciertos teóricos propios e incorporar el enfoque del punto de vista, para explicar y analizar dicho tema, considerando la perspectiva de las principales afectadas, las mujeres (Lau, 2002). Como hemos señalado, esta opción enriquece el conocimiento científico y facilita el desmontaje de los prejuicios culturales y técnicos contra los que se pronuncian las epistemologías feministas.

Recapitulando, la importancia de proponer como marco el enfoque del punto de vista feminista para indagar sobre las violencias en contra las mujeres, radica en la capacidad que tiene para relacionar a las personas concretas con su entorno y contexto social, estableciendo de manera estructurada las categorías analíticas que intervienen en la comprensión de las violencias de género. Esto, sin olvidar las relaciones subjetivas e intersubjetivas, que son parte fundamental en las epistemologías feministas $y$, acorde con los antecedentes que le dieron origen -los movimientos feministas-, no es ajena a la lucha por la igualdad entre hombres y mujeres; una aportación realmente imprescindible en el campo de las ciencias sociales.

\section{La categoría género desde la teoría feminista}

El término género dentro de la teoría feminista, se utiliza como categoría explicativa y como categoría analítica. En el primer caso sirve para discernir las múltiples construcciones y representaciones que se hacen sobre los cuerpos sexuados; y en el segundo, permite visibilizar las desigualdades sociales e indudablemente nos aproxima a develar las jerarquías sociales que mantienen posiciones de dominación-subordinación en la dinámica entre hombres y mujeres (De Barbieri, 1993). 
El sistema sexo/género, término incorporado por Gayle Rubin en 1975, define al género como una construcción cultural, establecida a partir de las diferencias biológicas, que determinadas como "diferencias sexuales" producen desigualdades, es decir, propician oportunidades diferenciadas para mujeres y hombres en la construcción de las relaciones sociales.

Los sistemas de género/sexo son los conjuntos de prácticas, símbolos, representaciones, normas y valores sociales que las sociedades elaboran a partir de la diferencia sexual anatómico-fisiológica y que dan sentido a la satisfacción de los impulsos sexuales, a la reproducción de la especie humana y en general al relacionamiento entre las personas (De Barbieri, 1993:150)

El sistema de sexo/género sirve para comprender las relaciones de dominación y subordinación femenina (Rubin, 1986) y, en consecuencia, las violencias contra las mujeres. La subordinación femenina permite entender que todo lo que se encuentre dentro del concepto de femenino tiende a ser parte de un sistema de opresión. Con la noción de feminidad se hace referencia a identidades, roles, símbolos y prácticas sociales que han sido determinados bajo este concepto. Este sistema, tal como lo menciona Teresita de Barbieri (1993), nos permite más posibilidades, para mirar las múltiples dimensiones de las relaciones entre hombres y mujeres, con base en lo que es considerado femenino o masculino y los significados sociales de ese orden binario que determina "dos modos de vida, dos tipos de subjetividad, de atributos eróticos, económicos, sociales, culturales, psicológicos, políticos, dos modos de sentir y de existir" (Fernández Rius, 2012: 80), y junto con este orden binario también se establece el ámbito público y privado, dando continuidad a una estructura que va de lo macro a lo microsocial y se instala en las subjetividades colectivas e individuales.

Por otra parte, el concepto de género da lugar a que las teorías feministas tengan un marco conceptual propio, para entender que las jerarquías y las relaciones de poder son dimensiones sociales y políticas, construidas sobre las diferencias anatómicas (Scott, 1996). En este sentido, el género como categoría de análisis, introducida por las feministas académicas desde la década de 1970, va a dar cuenta de las opresiones y subordinaciones que se viven en las relaciones de dominación.

En el caso específico de las mujeres, la dominación a la que nos hayamos subordinadas es multidimensional y puede presentarse en cualquier ámbito; sin embargo, es importante señalar que el ámbito privado-doméstico es en donde los estudios feministas comenzaron a definir la existencia de acciones de dominación, porque son espacios en donde las actividades se ven determinadas culturalmente por el sexo. La dicotomía entre lo privadopúblico vinculada a lo femenino y masculino respectivamente, constituye estereotipos y condiciona las maneras de actuar en cada sociedad (Lamas, 2013). Se entiende entonces, que estas condiciones del sexo y del género construyen sistemas simbólicos vinculados a las estructuras de dominación, donde las mujeres nos encontramos en una posición de subordinación naturalizada y determinada tanto social como culturalmente.

Rosa Cobo (2005) señala que el feminismo en su dimensión de tradición intelectual ha servido para mostrar la historicidad de los conocimientos, por tanto, cuando existen colectivos sociales que no están representados como sujetos de las investigaciones o que produzcan esas investigaciones, no puede haber objetividad científica. Por esa razón, cuando las mujeres comenzaron a irrumpir en las ciencias sociales se tambalearon los paradigmas establecidos con los que se hacían las investigaciones. Bartra (1999) distingue tres formas en las que teórica y epistemológicamente se han realizado estudios desde la academia, mismos que buscan develar las relaciones entre los géneros. Se trata, por un lado, de los estudios sobre mujeres cuyo centro de interés consiste en estudiar este colectivo y sus especificidades, en comparación con los hombres; otro bloque es el que se decanta por los estudios de género, explicando las diferencias culturalmente asociadas a cada sexo y la tercera vertiente, se refiere a los estudios feministas, mediante los que se denuncian las relaciones de poder, apelando y adscribiéndose a la voluntad política para eliminar las desigualdades.

En la confluencia de los movimientos feministas de 1970 -y décadas posteriores- el desarrollo de investigaciones académicas que van incorporando el concepto de género para señalar la existencia de oportunidades diferenciadas, se soportan en las diferencias sexuales entre las personas; aparece la demanda de justicia social hacia los poderes públicos, de los que se esperan intervenciones sociales y políticas centradas en erradicar las desigualdades entre hombres y mujeres, bajo la connotación de que lo privado es público, es decir, es cuestión del Estado (Amorós y De Miguel, 2005). Se diseñaron, en consecuencia, políticas públicas para la igualdad de género y los problemas que se consideraban privados como las violencias en los grupos familiares, salieron a la luz.

Como se ha planteado, los aportes de los feminismos, tanto desde el activismo como desde la academia, han trascendido en la búsqueda de mejoras para las mujeres en el plano internacional, cuyas demandas se han ido plasmando en documentos y legislaciones a favor de la 
igualdad entre los seres humanos. Como ejemplo, en la Conferencia Mundial sobre las Mujeres, realizada en Beijing, en 1995, se enunció la obligación de transversalizar la perspectiva de género en las políticas públicas, para incidir con sus acciones en todos los niveles y ámbitos de acción de los gobiernos, de manera coordinada.

En general, el género como categoría de análisis, ha revelado la existencia de las relaciones de poder y desarticulado las cuestiones culturales que en gran medida juegan en contra de las mujeres. Además, involucra entre otras a la clase, la etnia, la religión y la preferencia sexual, para definir la posición relativa de cada individuo o grupo. Por lo que respecta al análisis de las violencias contra las mujeres, la categoría género muestra que las sociedades construidas bajo la lógica de la dominación masculina permiten que las mujeres seamos vistas en una posición de inferioridad, sobre las que se ejerce el control y del poder. Nociones que son generadas bajo el orden binario, que se reproduce en los discursos y acciones, legitimando los espacios, ideologías y prácticas de poder y por ende, las violencias como método de opresión y sometimiento de las mujeres.

\section{Las desigualdades de género. Un problema de violencia estructural.}

Las teorías formuladas desde los feminismos abordan a las violencias contra las mujeres como consecuencia y como mecanismos del sistema patriarcal, que es alimentado por las construcciones culturales del género. Estas relaciones de poder que subyacen entre dichas construcciones, promueven las desigualdades y naturalizan las violencias que se desarrollan a través de las estructuras, los discursos y las manifestaciones simbólicas (Banchs, 1996). En este sentido, el concepto género, nos aproxima a observar la forma en que ocurre la reproducción social de las desigualdades, así como los ámbitos que propician, promueven, legitiman y naturalizan la dominación del poder masculino.

A pesar de que, en términos teóricos, la organización social patriarcal ha "mejorado" las condiciones de vida de las mujeres, con el desarrollo de algunas áreas de oportunidad, la realidad es que el patriarcado estructura las organizaciones de la vida social occidental y por supuesto, la latinoamericana. La trampa del aparente progreso es la que ha permitido al androcentrismo mantenerse hegemónicamente, sin dar cabida a su relevo por formas más democráticas en la organización de la sociedad. De este modo, se sigue reforzando el poder y las cargas culturales como el machismo, la fuerza, la dominación, la agresión o la misoginia, todas estas características son "propias" de las violencias contra las mujeres (Lagarde, 2013).

De modo que, dentro del sistema patriarcal, más allá de las violencias reconocidas, se encuentra un sistema social y cultural que legitima una violencia aún más profunda y peligrosa: la violencia estructural. Este concepto -siguiendo a Galtung (2003)- trata de identificar que si bien la violencia directa es una cuestión profundamente complicada y grave, no podría ser sostenida o llevada a cabo sin una violencia que se ejerce en ese otro campo y que no puede identificarse tan fácilmente. Así, la violencia estructural se instala en las identidades, las libertades y en las estructuras sociales, económicas, políticas y culturales que pueden o no generar conflictos. Aparece en toda esa base que propicia y produce una cierta forma de pensamiento, que sostiene un poder y dominación en todos los ámbitos sociales, creando discursos que legitiman las violencias contra las mujeres de una forma tal, que su reconocimiento es difícil de identificar.

Peor aún, en muchas ocasiones las violencias contra las mujeres son visibles y hasta justificadas por discursos de dominación-subordinación; sin embargo, a pesar de no ser acciones toleradas, existe una dinámica que permite naturalizarlas y mantener a las personas acríticas con las acciones que de ellas devienen, desde las más sutiles hasta las manifestaciones más brutales de opresión. Siguiendo el análisis realizado por Galtung (2003), la violencia cultural-simbólica también resulta una categoría importante, al entender las múltiples violencias contra las mujeres, ésta se presenta como la base ideológica de las violencias. En ella se mantienen las cuestiones simbólicas que legitiman, en clave de género, los roles masculinos y femeninos, mismos que promueven comportamientos e ideologías arraigadas a una jerarquización de dominación-subordinación.

En este sentido, hablar del sistema simbólico que se encuentra sosteniendo a la violencia cultural, permite también hacer un esbozo de la violencia simbólica, definida por Bourdieu (2000) como la lógica de poder y dominación que se ejerce con cierto consentimiento tanto de la parte dominada, como de la subordinada, determinada por un poder simbólico que define las maneras, acciones y representaciones que pueden darse en distintos estados de dominación, tal como lo es la dominación masculina. Esta violencia simbólica se ejerce de manera imperceptible e incluso tolerada, a través de discursos que normalizan y naturalizan la violencia estructural. Práctica que se convierte en el medio por el cual los estereotipos, roles y normas en torno a las condiciones del género subordinado son reforzadas ideológicamente, desembocando en actos de represión hacia el colectivo de las mujeres (Galtung, 2003). 
La violencia estructural al mantener el poder y la subordinación de los hombres hacia las mujeres, termina manifestándose de manera directa. Así, las violencias contra las mujeres, inician en la violencia cultural, teniendo como un detonante la violencia estructural, hasta concluir en la violencia física (Magallón, 2005). Tomando en consideración lo anterior, no es posible abordar las violencias contra las mujeres fuera de una violencia estructural, que, como ya se dijo es una práctica que se encuentra arraigada por sistemas de dominación ideológicos patriarcales, que son resultado de procesos y estructuras conformadas a partir de la construcción social del género.

De esta manera, para comprender las violencias más allá de lo evidente, se debe tomar en cuenta que la violencia estructural juega un papel determinante en las dinámicas de poder, por lo que es necesario hacer un esfuerzo por develar las formas en que se han naturalizado las desigualdades en todos los ámbitos presentes en la vida de cada mujer, hasta las manifestaciones de los aspectos simbólicos que devienen de la construcción social del género a partir de la diferencia anatómica del sexo. Sin olvidar que la violencia directa es la representación interiorizada de una estructura que no permite el incumplimiento de las normas del orden social ya establecido. Por el contrario, se mantiene aferrada a la preservación del sistema social y cultural que propicia las jerarquías, mismas que constituyen factores de riesgo por las formas de dominación que solapan, afectando la integridad y la seguridad que por la condición de ser mujeres estamos expuestas a vivir.

Siguiendo la aproximación teórica feminista planteada, para el estudio de las violencias en contra de las mujeres, develar la violencia estructural es un paso necesario en el análisis global de las violencias vividas individualmente, porque permite entender las condiciones del contexto en el que se producen. Recordar que el punto de vista feminista como epistemología y la categoría de género, buscan poner de manifiesto las experiencias y subjetividades de cada persona, sin olvidar las realidades y variables que se interseccionan en cada vida, porque son elementos que enriquecen el estudio de un problema complejo, que nos invita a mirar más allá de lo evidente.

\section{Consideraciones finales}

Las violencias como ejercicios de poder reiteran la capacidad de opresión, que en el caso particular de las relaciones entre hombres y mujeres giran en torno a la desigualdad, la discriminación, la subordinación y la agresión. Estas dimensiones de la violencia contra las mujeres, van más allá de los actos físicos y forman parte de situaciones conflictivas que en todo momento se ven intersectadas por otros elementos como la etnia, la clase social y otras condicionantes de pertenecer a una minoría, que se suman al género y las construcciones socioculturales que éste implica.

Se ha planteado que para comprender parte de este problema, se deben identificar las relaciones de dominación presentes en los ámbitos sociales y sus manifestaciones, reconocer también las situaciones desiguales presentes en muchos aspectos de la vida individual y colectiva de las mujeres, así como tener en cuenta que investigar sobre las violencias en contra de las mujeres requiere de un profundo ejercicio de reflexión para desarticular todas esas manifestaciones invisibles que se encuentran "ocultas" en la violencia estructural.

Como ya se ha advertido, las relaciones de poder están presentes en diferentes espacios cotidianos, públicos y privados, y las construcciones culturales de los géneros nos han encaminado a un orden binario dentro de la estructura social, donde lo femenino pareciera ser, en sí misma, una cuestión determinante en la construcción de la violencia contra las mujeres.

La complejidad del estudio sobre las violencias en contra de las mujeres y sus múltiples dimensiones, también nos obliga a hacer una revisión interdisciplinar y consiente de los privilegios como estudiosas del tema. Realizar estos acercamientos es parte del quehacer desde una mirada académica feminista, que contribuirá a deconstruir los mecanismos de dominación que estamos reproduciendo tanto en los espacios públicos como en los privados, con la intención de generar transformaciones de manera pertinente, en nuestras relaciones con las demás personas.

Los marcos teóricos e interpretativos, como los que devienen de las epistemologías feministas, particularmente el punto de vista feminista, facilitan el acercamiento a la realidad social de las violencias, con el fin de entender la complejidad del problema. Construir el conocimiento a partir de este enfoque facilita el reconocimiento, la comprensión y el cuestionamiento de manera crítica de todas las categorías implicadas en el estudio de las mujeres.

Frente a problemas de gran magnitud, como lo son las violencias contra las mujeres, estamos obligadas a profundizar en su estudio a nivel microsocial, para develar las múltiples conformaciones que las mantienen $\mathrm{y}$, en todo caso, para vincular a las mujeres y hombres en una lucha por un mundo más igualitario y justo, materializando las acciones para los cambios a partir de fundamentos como los que proveen las epistemologías feministas y las nociones sobre el género y la violencia estructural. 


\section{Referencias Bibliográficas}

Amorós, Celia y De Miguel, Ana (2005) Teoría feminista de la ilustración a la globalización. 3 volúmenes. Madrid, España: Ediciones Minerva.

Araiza, Alejandra (2012) De la política de la localización a los conocimientos situados. Notas para la creación de una ciencia feminista. En M. Liévano y M. Duque (Comp.) Subjetivación femenina: Investigación, estrategias y dispositivos críticos, pp.163-191. Monterrey, México: Universidad Autónoma de Nuevo León.

Araiza, Alejandra (2017) Ciencia, subjetividad y poder. Pachuca, México: UAEH.

Banchs, María. A. (julio, 1996) Violencia de género. Revista Venezolana de Análisis de Coyuntura, 2(2), pp.11-23.

Bartra, Eli (1999) El movimiento feminista en México y su vínculo con la academia. Revista de Estudios de Género. La Ventana, (10), pp. 214 234

Bartra, Eli (2012) Acerca de la investigación y la metodología feminista. En Norma Blázquez, Fátima Flores y Maribel Ríos (Coord.) Investigación feminista. Epistemología feminista, metodología y representaciones sociales, pp. 67-78. Ciudad de México, México: Universidad Nacional Autónoma de México.

Beltrán, Elena y Maquieira, Virginia (2001) Feminismos. Debates teóricos contemporáneos. Madrid, España: Alianza Editorial.

Biglia, Bárbara (2007) Resignificando violencia(s), obra feminista en tres actos y un falso epílogo. En Bárbara Biglia, y Conchi San Martín (Coord.) Estado de Wondebra. Entretejiendo narraciones feministas sobre las violencias de género, pp. 21- 34. Barcelona, España: Lallevir.

Blázquez, Norma (2012) Epistemología feminista: Temas centrales. En Norma Blázquez, Fátima Flores y Maribel Ríos (Coord.) Investigación feminista. Epistemología feminista, metodología y representaciones sociales, pp. 21-38. Ciudad de México, México: Universidad Nacional Autónoma de México.

Bourdieu, Pierre (2000) La dominación masculina. Barcelona, España: Anagrama.

Cobo Bedia, Rosa (2005) El género en las Ciencias Sociales. Cuadernos de Trabajo Social $18 . \quad$ Recuperado de: http://revistas.ucm.es/index.php/CUTS/article/view/CUTS05051102 $\underline{49 \mathrm{~A} / 755}$

De Barbieri, Teresita (1993) Sobre la categoría de género. Una introducción teórica metodológica. Debates en sociología (18), pp. 145-169. Ciudad de México, México: Universidad Nacional Autónoma de México.

DOF (1 de febrero de 2007) Ley General de Acceso de las Mujeres a un Vida Libre de Violencia. Diario Oficial de la Federación Última reforma 13042018 México: Secretaría de gobernación. Recuperado de: http://www.diputados.gob.mx/LeyesBiblio/pdf/LGAMVLV 130418. pdf

European Commission: Research \& Innovation (2011) Manual El género en la investigación. Comisión Europea: Unidad de Mujeres y Ciencia del Ministerio de Ciencia e Innovación.

Fernández Rius, Lourdes (2012) Género y ciencia. Entre la tradición y la transgresión. En Norma Blázquez, Fátima Flores y Maribel Ríos (Coord.) Investigación feminista. Epistemología feminista, metodología y representaciones sociales, pp. 70-110. Ciudad de México, México: Universidad Nacional Autónoma de México.

Galtung, Johan. (2016) La violencia: cultural, estructural y directa. Cuadernos de estrategias (183), pp. 147-168. Recuperado de https://dialnet.unirioja.es/ejemplar/451439 [13 de febrero de 2019].

Harding, Sandra (1996). Ciencia y feminismo. Madrid, España: Morata.

Harding, Sandra (2002) ¿Existe un método feminista? En E. Bartra (Comp.) Debates en torno a una metodología feminista, pp. 9-34. Ciudad de México, México: Universidad Autónoma Metropolitana.
Lagarde, Marcela (2013) Metodologías Feministas para la formación de mujeres líderes en M. P. Castañeda (Coord.) Perspectivas feministas para fortalecer los liderazgos de mujeres jóvenes, pp. 23-90. Ciudad de México, México: Centro de Investigaciones Interdisciplinarias en Ciencias y Humanidades Universidad Nacional Autónoma de México.

Lamas, Marta (2013) Usos, dificultades y posibilidades de la categoría género. En Marta Lamas (Comp.) El género. La construcción cultural de la diferencia sexual, pp. 327-366. Ciudad de México, México: Universidad Nacional Autónoma de México.

Lau Jaiven, Ana (2002) Cuando hablan las mujeres. En Eli Bartra (Comp.) Debates en torno a una metodología feminista, pp. 185-198. Ciudad de México, México: Universidad Autónoma Metropolitana.

Maddo Lengerman, Patricia y Niebrugge-Branlley, Jill (1997) Teoría feminista contemporánea. En George Ritzer (Coord.) Teoría Sociológica contemporánea, pp. 353-409. Ciudad de México, México: McGraw Hill.

Magallón Portolés, Carmen (2005) Epistemología y violencia. Aproximación a una visión integral sobre la violencia hacia las mujeres. En Feminismos (6), pp. 33-47. Recuperado de: https://rua.ua.es/dspace/bitstream/10045/3165/1/Feminismos 6 03.p df

Martín Casares, Aurelia (2008) Antropología del género. Culturas, mitos y estereotipos sexuales. Madrid, España: Ediciones Cátedra.

Meza Mejía, Sofía (2018) Interseccionalidad. En Jorge Ramírez Plascencia (Coord.), Conceptos claves en Ciencias Sociales. Definición y aplicaciones, pp. 235-256. Guadalajara, México: Universidad de Guadalajara.

Rubin, Gayle (1986) El tráfico de mujeres: notas sobre la "economía política" del sexo. En Nueva Antropología, 3(30), pp. 95-145.

Scott, Joan W. (1996) El género, una categoría útil para el análisis histórico. En Marta Lamas (Comp.) El género: la construcción cultural de la diferencia sexual, pp. 265-302. México, Ciudad de México: Programa Universitario de Estudios de Género-Universidad Nacional Autónoma de México. 